\title{
Effect of C-factor and LED Curing Mode on Microleakage of Class V Resin Composite Restorations
}

\author{
Qasem Diefallah Alomaria \\ Kefah Barrieshi-Nusair ${ }^{b}$ \\ Mohammad Alic
}

\begin{abstract}
Objectives: To investigate the effects of cavity C-factor and LED curing mode on microleakage of class $\mathrm{V}$ resin composite restorations.

Methods: Eighty extracted human maxillary premolars were divided into four groups. In groups I and II, V-shaped class $V$ cavities ( $C$-factor $\approx 1.5$ ) were prepared while box-shaped class $V$ cavities (high $\mathrm{C}$-factor $\approx 4$ ) were prepared in groups III and IV. All the cavities were prepared on the vestibular surfaces of the teeth. All the cavities were prepared by one operator with specific dimensions. The same adhesive system (Excite) and the same restorative composite (Tetric Ceram) were used in all the groups. The restorations in groups I and III were cured with the fast curing mode of LED curing light, while the restorations in groups II and IV were cured with the soft-start mode of the same curing light. The samples were thermocycled, immersed in a $0.5 \%$ basic fuchsin solution for 24 hours, and sectioned. The degree of dye penetration was measured quantitatively and then qualitatively at both enamel and dentin/cementum margins.

Results: Quantitatively, there was no statistical difference in the degree of microleakage between the groups at the enamel margin ( $P>.05)$. At the dentin/cementum margin, group III showed significantly more dye penetration than the other groups $(P<.001)$. Similarly, qualitative measurements of dye penetration showed that group III had higher microleakage at dentin/cementum margin than the remaining groups (I and III P<.001; II and III P<.01; III and IV P<.01). Paired sample statistics showed that microleakage at the dentin/cementum margin was not statistically different from enamel microleakage $(P>.05)$, except for group III $(P<.000)$.

Conclusions: Fast curing mode of LED curing light resulted in more microleakage at the gingival floor of class $\mathrm{V}$ cavities with high $\mathrm{C}$-factor. There was no difference in dye penetration between enamel and dentin margins of the cavities except when the fast curing mode was used with high $\mathrm{C}$-factor cavities. Both quantitative and qualitative measurements of microleakage gave the same results at both enamel and dentin margins. (Eur J Dent 2011;5:400-408)
\end{abstract}

Key words: Resin composite; Microleakage; C-factor; LED curing units; Curing modes.

Department of Restorative Sciences, Faculty of Dentistry, Kuwait University, Kuwait.

b Deceased.

c Department of Diagnostic Sciences, Faculty of Dentistry, Kuwait University, Kuwait.
Corresponding author: Qasem Diefallah Alomari Department of Restorative Sciences, Faculty of Dentistry, Kuwait University, P. O. Box 24923, Alsafat 13110, Kuwait. E-mail: qalomariahsc.edu.kw qasemalomari70ayahoo.com 


\section{INTRODUCTION}

One major drawback of resin composite restorative materials is the polymerization shrinkage caused by the dimensional rearrangement of monomers into polymer chains during polymerization reaction. ${ }^{1}$ Clinically, the polymerization shrinkage is restrained by the developing bond of the restorative material to the cavity walls. This restriction induces polymerization shrinkage stress, which counteracts the developing resintooth bond by pulling the setting resin composite material away from the cavity walls. Failure of the bond at the tooth-resin interface will result in microleakage and postoperative sensitivity. ${ }^{2,3}$ In addition, it was shown that polymerization shrinkage stress tremendously weakens the performance and longevity of dental composites. ${ }^{4}$ Numerous approaches have been proposed to minimize the shrinkage stress by manipulation of curing protocols and placement techniques. ${ }^{5}$ Among these approaches, soft-start curing (which can be either in a step or a ramp mode) and pulse curing have attracted extensive investigations. ${ }^{6-11}$ The hypothesis for advocating these curing protocols is that the initial low-light intensity could facilitate a certain degree of shrinkage stress relaxation before the system reaches the vitrification stage and while in the pre-gel phase. Laboratory research showed that soft-start polymerization partially relieves shrinkage stress and achieves improved marginal integrity of the restoration. ${ }^{10}$ On the other hand; some researchers have showed that the stress relief advantage of soft-start curing might be in fact a result of reduction in the degree of conversion of the resin. ${ }^{11-13}$

Previous studies have shown that polymerization stress magnitude is influenced by the characteristics of the cavity to be restored..$^{14-17}$ The cavity configuration or $\mathrm{C}$-factor is defined as the ratio of the bonded to the unbonded surface area. ${ }^{17}$ During light-induced polymerization of resin composite, the shrinkage forces in high $\mathrm{C}$-factor cavities cannot be relieved by resin flow, resulting in the debonding of one or more walls. ${ }^{14-19}$ Using different adhesive systems, it has been found that the C-factor of the cavity negatively affect the microtensile bond strength to dentin. ${ }^{20}$

In addition to conventional halogen-based light activation units, light-emitting diode (LED) has been introduced for the polymerization of resin composite restorations. LEDs hold several advantages over halogen-based units, including having extended lifetimes, more light efficiency, not requiring filters, and having higher resistance to shock and vibration. ${ }^{9,21}$

No studies have yet examined the combined effects of C-factor and soft-start mode of LED curing light on microleakage of class $\mathrm{V}$ resin composite restorations. Therefore, the objective of this study was to compare the amounts of microleakage observed when using two different modes of LED curing light (fast and soft-start) for two designs of class $V$ cavity preparation: a V-shaped cavity design and a box-shaped cavity design. The hypothesis tested was that combining the effect of fast curing mode and high $\mathrm{C}$-factor cavity will not affect the degree of microleakage around class $\mathrm{V}$ resin composite restorations.

\section{MATERIALS AND METHODS}

Eighty freshly extracted non-carious human maxillary premolar teeth were used in the study. After extraction, the teeth were cleaned, disinfected in $10 \%$ formalin for two weeks and then stored in physiological saline. Only undamaged teeth were used. To ensure that no dye would reach the cavities via the pulp chamber and the dentinal tubules, the apical foramina were sealed as follows. The root apices were cut off; then, a cavity was prepared at the cut root apices with an inverted cone carbide bur (Meisinger, Hager \& Meisinger $\mathrm{GmbH}$, Germanyl and restored with glass ionomer restorative material (Fuji II LC, GC Corporation, Tokyo, Japan).

The teeth were randomly divided into four equal groups (Table 1). In groups I and II, V-shaped class $\mathrm{V}$ cavities were prepared on the vestibular surfaces of the teeth, using straight fissure carbide bur, size 57 (American Numbering system) (DENTSPLY International, 221 W. Philadelphia Street, York, PA 17405-0872; USA; Lot \# 401809) at high speed handpiece and with water-cooling. The bur was replaced every five cavities. The cavities were prepared in enamel and dentin at the cemento-enamel junction (CEJ). The deepest part of the cavities was at the CEJ (2 $\mathrm{mm}$ deep). The coronal slope was in enamel and ended at a 2 $\mathrm{mm}$ distance from the CEJ. The apical slope was in dentin and ended at a $2 \mathrm{~mm}$ distance from the 
CEJ. The mesio-distal dimension of the cavities was $4 \mathrm{~mm}$ (Figures $1 \mathrm{a}$ and $1 \mathrm{~b}$ ). The cavities were standardized for depth by a mark on the burs and for the diameter by placing a sticker with a $4 \mathrm{~mm}$ punched hole on the desired area. ${ }^{22}$ The resulting $\mathrm{C}$-factor for the cavities was calculated as the ratio of the bonded to the unbonded surface area of the cavity. The cavities in these groups were visualized approximately as a triangular prism. The surface area of a triangular prism $=[a b+l s 1+s 2$ + s3) h] ( $a=$ altitude of the triangle, $b=$ base of the triangle, $\mathrm{s}=$ side of the triangle, and $\mathrm{h}=$ height of the prism $]=[2 \times 4+(2+2+4) 4]=40 \mathrm{~mm}^{2}$. The unbonded surface area of the cavity is visualized as a rectangle, therefore its surface area $=$ length $\mathrm{x}$ width $=[4 \times 4]=16 \mathrm{~mm}^{2}$. The bonded surface area $=$ the surface area of the prism - the unbonded surface area $=40-16=24 \mathrm{~mm}^{2}$. Therefore, the resulting $\mathrm{C}$-factor $=24 / 16=1,5$.

In groups III and IV, box-shaped class V cavities were prepared on the vestibular surfaces of the teeth using fissure carbide burs at high speed handpiece and water coolant. The cavities were prepared in enamel and dentin at the CEJ. The dimensions of the cavities were: $2 \mathrm{~mm}$ in depth, $4 \mathrm{~mm}$ mesio-distal in width, and $2 \mathrm{~mm}$ occluso-gingival in height (Figures $2 \mathrm{a}$ and $2 \mathrm{~b}$ ). The cavities were visualized approximately as a rectangular prism. The surface area of the rectangular prism $=[2(l w)+(2 l+2 w) h](l=$ length of the prism, $\mathrm{w}=$ width of the prism, and $\mathrm{h}=$ height of the prism $)=[2(2 \times 2)+(2 \times 2+2 \times 2) \times 4]=40 \mathrm{~mm}^{2}$. The unbonded surface area of the cavity is visualized as a rectangle, therefore its surface area $=$ length $x$ width $=[4 \times 2]=8 \mathrm{~mm}^{2}$. The bonded surface area of the cavity $=$ the surface area of the prism - the unbonded surface area $=40-8=32$ $\mathrm{mm}^{2}$. Therefore, the resulting $\mathrm{C}$-factor $=32 / 8=4$.

The volume of the V-shaped cavities (the triangular prism) was calculated using the following formula $[1 / 2 a b h]=[1 / 2(2 \times 4 \times 4)]=16 \mathrm{~mm}^{3}$. The volume of the box-shaped cavities (rectangular prism) was calculated based on the following formula $[\mathrm{lwh}]=2 \times 2 \times 4=16 \mathrm{~mm}^{3}$. The volumes of the cavities were equal for all groups, namely 16 $\mathrm{mm}^{3}$.

One curing unit (Mini L.E.D, Satelec, Merignac, Cedex, France) with a curing guide of $7.5 \mathrm{~mm}$ diameter was used throughout the study. All the cavities were treated with $35 \%$ phosphoric acid for 15 seconds. After removal of all etchant gel with a vigorous water spray for 10 seconds, excess water was removed with high volume evacuation tip placed directly over the preparation for 1-2 seconds. Then, the dentin bonding agent (Excite, Ivoclar Vivadent, Bendererstrasse 2, FL9494 Schaan, Liechtenstein) was applied to both enamel and dentin with a disposable brush, according to manufacturer instructions. To obtain a uniform, glossy appearance and solvent free adhesive, a high-volume evacuation tip was placed over the preparation for 1-2 seconds, and then the adhesive was light cured for 15 seconds. The cavities were then filled with microhybrid resinbased composite material (Tetric Ceram, shade A3, Ivoclar Vivadent, Bendererstrasse 2, FL-9494 Schaan, Liechtenstein) in one increment.

The resin composite in groups I and III was cured for 15 seconds using the fast curing mode of the LED curing device (light intensity $1100 \mathrm{~mW}$ / $\mathrm{cm}^{2}$ ). On the other hand, the resin composite in groups II and IV was cured for 20 seconds, using soft-start curing for 10 seconds from 0 to 1100 $\mathrm{mW} / \mathrm{cm}^{2}$, followed by $1100 \mathrm{~mW} / \mathrm{cm}^{2}$ for $10 \mathrm{sec}-$ onds. The two curing modes resulted in the same radiation energy of $16.5 \mathrm{~J} / \mathrm{cm}^{2}$.

Immediately after curing, each restoration was contoured using ET finishing diamonds (Brasseler USA, Savannah, GA, USA), and then, specimens were placed in distilled water at $37^{\circ} \mathrm{C}$ for 24 hours, after which they were thermocycled 1000 times at $5^{\circ} \mathrm{C}$ and $55^{\circ} \mathrm{C}$ with a dwell time of 30 seconds. With the exception of $1 \mathrm{~mm}$ around the restoration, the entire tooth was covered with two layers of nail varnish. Specimens were placed in a solution of $0.5 \%$ basic fuchsin dye for 24 hours, after which they were removed, rinsed with distilled water, and embedded in autopolymerizing acrylic resin.

The embedded specimens were sectioned longitudinally in the bucco-lingual direction into halves using a water-cooled, slow-speed diamond saw (ISOMET, Buehler Ltd, Lake Bluff, IL, USA) and a diamond blade (15HC, Buehler Ltd, Lake Bluff, IL, USA). Pictures of the sections were taken with a digital camera (Leica DC 200, Leica Microsystems, Wetzlar GmbH, Ernst-LeitzStrasse, D-35578 Wetzlar, Germanyl attached to a stereomicroscope (Leica MZ-6, Leica Microsystems, Wetzlar GmbH, Ernst-Leitz-Strasse, 
D-35578 Wetzlar, Germanyl at magnification of $x 10$. The degree of dye penetration was measured quantitatively with an image analysis program (Leica IM 500, Leica Microsystems, Wetzlar GmbH, Ernst-Leitz-Strasse, D-35578 Wetzlar, Germany) with an accuracy of $0,001 \mathrm{~mm}$. Using the same pictures, qualitative dye penetration scores were taken separately according to the following criteria: $8,22-27$

$0=$ no dye penetration

1 = penetration of the dye to one-half of the cervical floor

2 = penetration of the dye along the entire length of the cervical floor

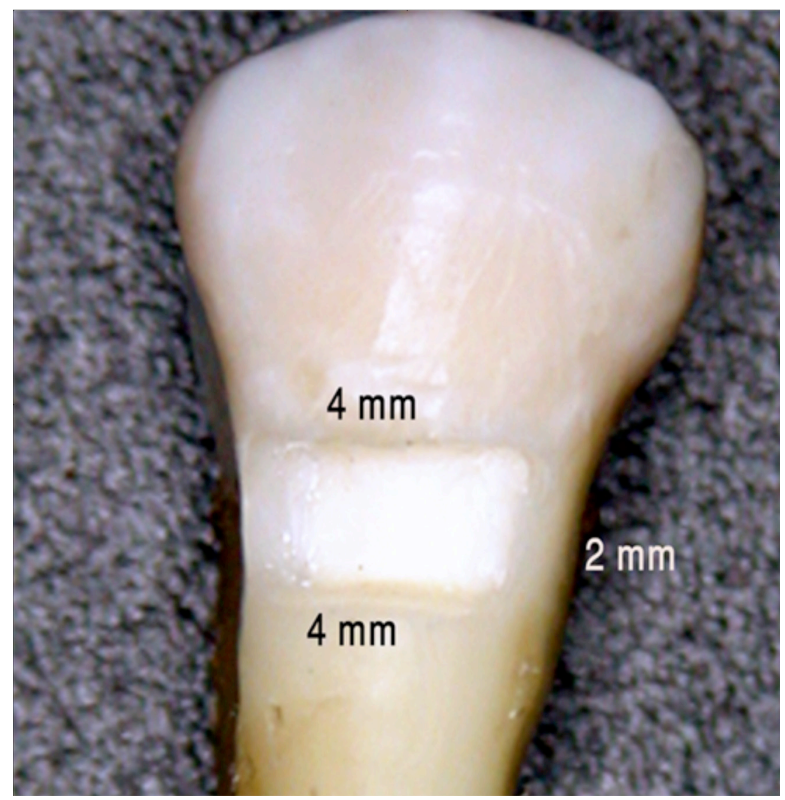

Figure 1a. Photograph of box-shaped class V cavity.

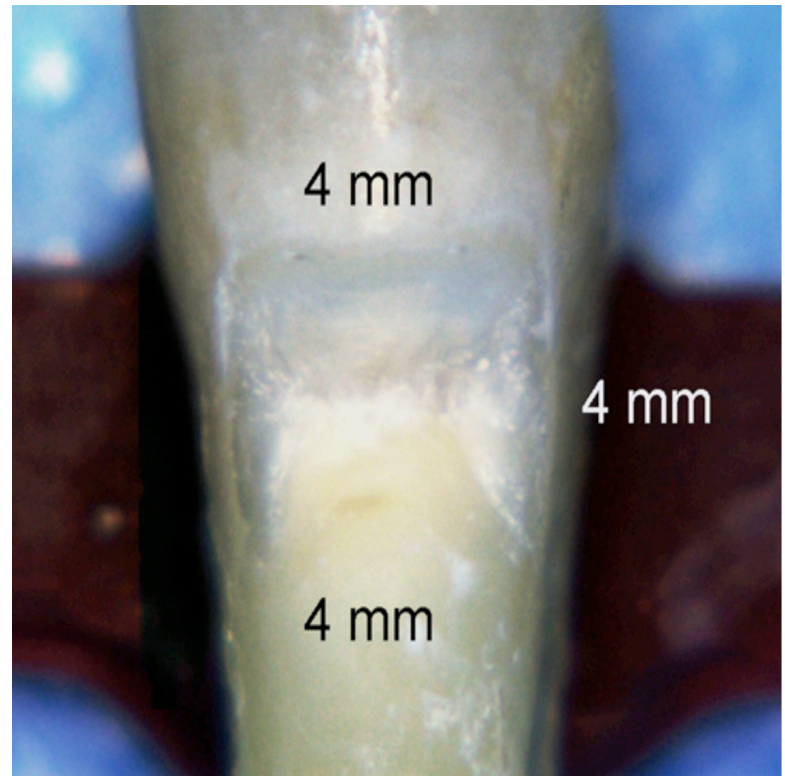

Figure 2a. Photograph of V-shaped class V cavity.
$3=$ penetration of the dye up to one-half of the length of the axial wall (in groups III and IV) or up to one-half of the length of the occlusal wall (in groups I and II)

$4=$ penetration of the dye greater than one-half of the axial wall (in groups III and IV) or greater than one-half of the length of the occlusal wall (in groups I and II)

Both the quantitative and qualitative measurements were taken by two examiners independently and at two different occasions. Prior to the investigation, calibration of both examiners was undertaken by reading 8 pilot samples which were not included in the study. Kappa value of 0.9 was

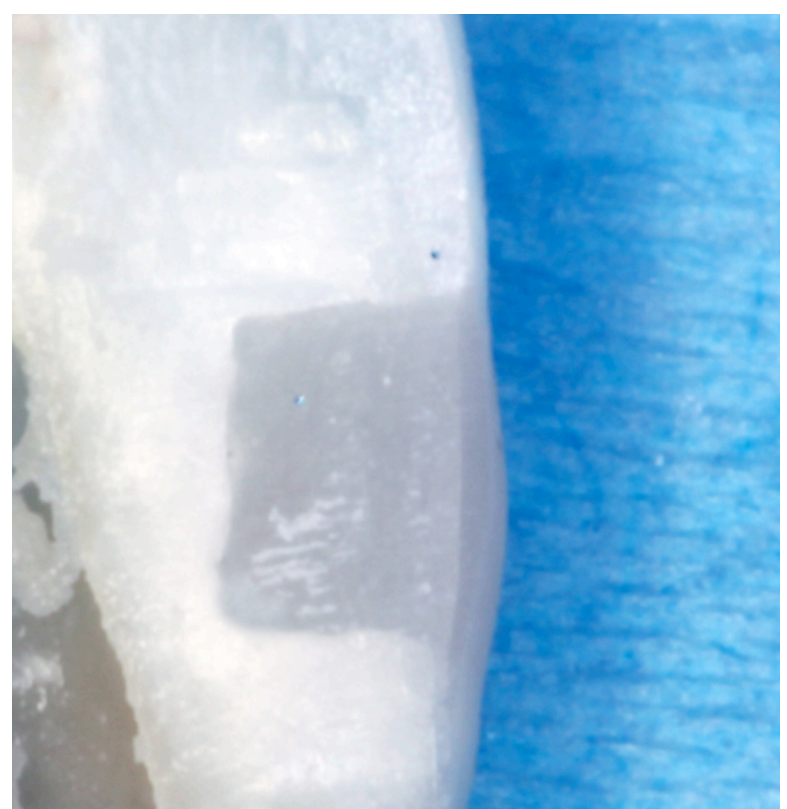

Figure 1b. Photograph of cross section of restored box-shaped class V cavity.

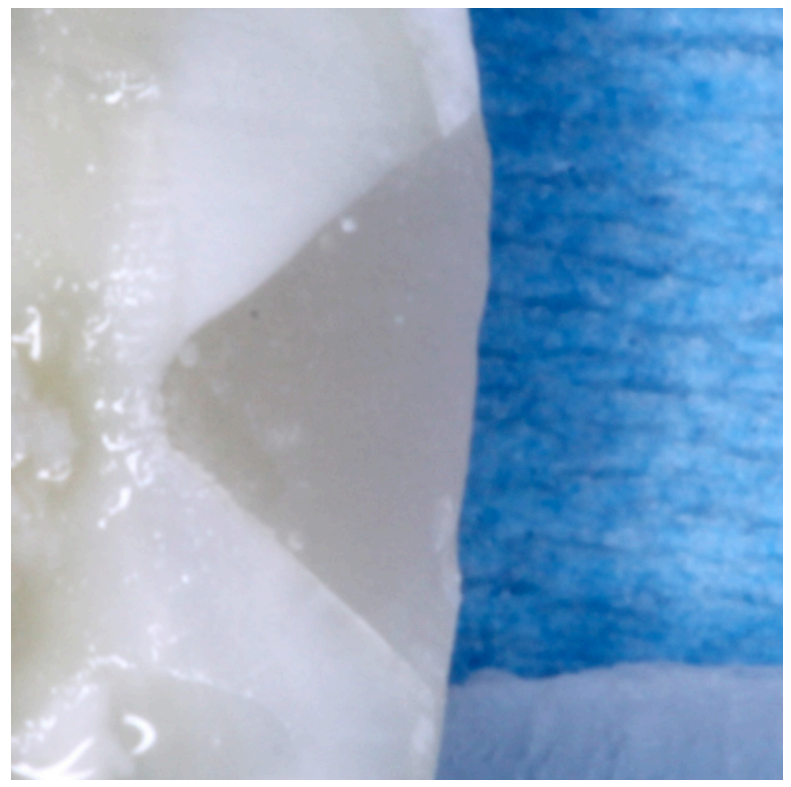

Figure 2b. Photograph of cross section of restored V-shaped class V cavity. 
achieved. For quantitative measurements, the average of the readings was taken, while for the qualitative measurements, the worst degree of dye penetration on both enamel and dentin margins were recorded.

Statistical analysis was performed using SPSS for Windows Version 16.0 (SPSS Inc., Chicago, IL). For the quantitative data, multivariate ANOVA was performed with two independent variables (cavity shape and curing mode), and with dye leakage at the enamel and at the cementum/dentine margins as dependent variables. Duncan's multiple range tests compared the groups at a significance level of $\alpha<.05$. For the qualitative data, the KruskalWallis test and the Mann-Whitney U-tests were used at a significance level of $\alpha<.05$. Paired sample statistics was used to compare microleakage at enamel and dentin margins. The significance level was set at 0.05 .

\section{RESULTS}

The results of the multivariate ANOVA on the effects of cavity shape and curing mode on dye penetration at the enamel and cementum/dentine margins are presented in Table 2. At the enamel margin, there were no observed effects of cavity shape and curing mode on dye penetration ( $P>$.05). On the other hand, at the cementum/dentine mar- gin, cavity shape and curing mode impacted dye leakage at the cementum/ dentin margin $(P<.001)$.

The mean depth of dye penetration for each group is reported in Table 3. Group III (box-shaped cavity with fast curing model was different than the other three groups and showed the highest mean for the depth of dye penetration. There was no statistical difference between the other three groups.

Table 4 shows the enamel dye penetration scores for the four groups. There was no statistical difference between the groups $(P>$.05). The predominant score for dye penetration in all groups was 0 (no leakage).

Table 5 shows the counts of dentin/cementum dye penetration scores. Only group III (boxshaped cavity with fast curing mode) was significantly different than the other three groups (I and III $P<.001$; II and III $P<.01$; III and IV $P<.01$ ). There was no statistical difference between the other groups ( $P>$.05).

Paired sample statistics was used to compare microleakage at enamel and dentin margins. There was no statistical difference in microleakage between enamel and dentin margins for all of the groups except for group III, where dentinal leakage was greater than enamel leakage $(P<.000)$.

Table 1. Distribution of the groups used in the study.

\begin{tabular}{lccccc}
\hline Group & N & Cavity shape & Curing mode & Radiation protocol & Radiation exposure \\
\hline I & 20 & V & Fast & 15 seconds at $1100 \mathrm{~mW} / \mathrm{cm}^{2}$ & $16.5 \mathrm{~J} / \mathrm{cm}^{2}$ \\
II & 20 & V & Soft-start & 10 seconds from 0 to $1100 \mathrm{~mW} / \mathrm{cm}^{2}+10$ seconds at $1100 \mathrm{~mW} / \mathrm{cm}^{2}$ & $16.5 \mathrm{~J} / \mathrm{cm}^{2}$ \\
III & 20 & Box & Fast & 15 seconds at $1100 \mathrm{~mW} / \mathrm{cm}^{2}$ & $16.5 \mathrm{~J} / \mathrm{cm}^{2}$ \\
IV & 20 & Box & Soft-start & 10 seconds from 0 to $1100 \mathrm{~mW} / \mathrm{cm}^{2}+10$ seconds at $1100 \mathrm{~mW} / \mathrm{cm}^{2}$ & $16.5 \mathrm{~J} / \mathrm{cm}^{2}$ \\
\hline
\end{tabular}

Table 2. Results for the multivariate ANOVA on the effect of cavity shape and curing mode on the dye penetration at the enamel and cementum/dentine.

\begin{tabular}{lccc}
\hline & & Multivariate test (Wilks' Lambda) & P-value \\
\hline Enamel Margin & F & df & ns \\
Cementum/Dentin Margin & 0.313 & 76 & $* * *$ \\
Enamel Margin X Cementum/Dentin Margin & 6.745 & 150 & $* * *$
\end{tabular}

Note: ${ }^{* * *} \mathrm{P}=.001 ; * * * \mathrm{P}=.0001$

Table 3. Means and standard deviations $(\mathrm{mm})$ of depth of dye penetration (measured quantitatively) at the occlusal and gingival margins.

\begin{tabular}{lcc}
\hline Group & Enamel margin & Cementum/Dentin margin \\
\hline I & $0.265(0.524)^{\mathrm{a}}$ & $0.485(0.731)^{\mathrm{a}}$ \\
II & $0.415(0.798)^{\mathrm{a}}$ & $0.670(0.850)^{\mathrm{a}}$ \\
III & $0.465(0.743)^{\mathrm{a}}$ & $1.400(0.538)^{\mathrm{b}}$ \\
IV & $0.420(0.688)^{\mathrm{a}}$ & $0.650(0.633)^{\mathrm{a}}$ \\
\hline
\end{tabular}

* Means for homogeneous subsets are displaced. 


\section{DISCUSSION}

According to the results of this study, the nullhypothesis that combining the effect of fast curing mode and high $\mathrm{C}$-factor cavity do not affect the degree of microleakage was rejected. When the restoration of a class $\mathrm{V}$ cavity with a high $\mathrm{C}$-factor was cured with the fast-curing mode, this resulted in more microleakage at the gingival margins.

Microleakage evaluation is the most common method of assessing the sealing efficiency of a restorative system. ${ }^{27}$ Class $V$ cavities located at the CEJ of the vestibular surfaces of maxillary premolars were used in this study. All the cavities were prepared and restored by one investigator following strict protocol. The adhesive system used (Excite) is a filled, light-curing, single component and alcohol based type of bonding agent. In a previous study, this adhesive system showed microtensile bond strength values of over $30 \mathrm{MPa}$ under both dry and moist conditions. ${ }^{28}$ After removal of all etchant gen with a vigorous water spray for 10 seconds, excess water was removed and dentin surfaces were left moist. Using one adhesive system and one resin composite restorative material reduced the confounding variables in the study.

Previous studies showed no effect of the insertion technique on microleakage of class $\mathrm{V}$ cavities. ${ }^{15,16}$ On the contrary, another study found that the incremental placement technique reduces microleakage when compared with the bulk placement technique in deep cavities. ${ }^{27}$ Furthermore, it has been found that $\mathrm{C}$-factor and placement technique can affect the microtensile bond strength of the adhesive system. ${ }^{20}$ In our study, to rule out the effect of the placement technique, the restorative material was placed as one increment because the depth of the cavities in all of the groups was only $2 \mathrm{~mm}$.

To simulate thermal stresses on the tooth-restoration interface, microleakage studies usually employ thermocycling of different regimens. ${ }^{22-26}$ One microleakage study showed that microleakage of resin composite restorations will not be affected by thermocycling, when the restorations are cured with soft-start polymerization of LED curing light. ${ }^{23}$ On the other hand, the same study showed a difference in microleakage between thermocycled and non-thermocycled specimens when standard and high intensity curing modes were used. For standardization, in our study, we used one protocol of thermocycling for all the groups.

To change the $\mathrm{C}$-factor for the cavities, two different shapes of cavities were prepared, namely the $V$ and box shapes. The sizes and surfaces available for bonding were the same for the two shapes of the cavities $116 \mathrm{~mm}^{3}$ and $\sim 32 \mathrm{~mm}^{2}$ respectivelyl. Previous studies showed contradictory results regarding the effect of $\mathrm{C}$-factor on composite resin restorations. Laboratory studies showed that high $\mathrm{C}$-factor increases the rate and amount of stresses resulting from polymerization shrinkage of resin composite restorations. ${ }^{19,29}$ Santini et $\mathrm{al}^{30}$ found no difference in the amount of microleakage between box-shaped cavities and $V$-shaped cavities at both enamel and gingival margins. Using bovine incisors, a difference

Table 4. Frequency (percentages) of enamel microleakage scores for the four groups. Kruskal-Wallis test value $=0.735$.

\begin{tabular}{|c|c|c|c|c|c|}
\hline Group & 0 & 1 & 2 & 3 & 4 \\
\hline 1 & $16(80)$ & 1 (5) & 1 (5) & $2(10)$ & $0(0)$ \\
\hline II & 15 (75) & 1 (5) & $0(0)$ & $1(5)$ & $3(15)$ \\
\hline III & $14(70)$ & $0(0)$ & $0(0)$ & $3(15)$ & $3(15)$ \\
\hline IV & $14(70)$ & $1(5)$ & $0(0)$ & $1(5)$ & $4(20)$ \\
\hline Total & $59(74)$ & $3(4)$ & $1(1)$ & 7 (9) & 10 (12) \\
\hline
\end{tabular}

Table 5. Frequency (percentages) of dentine/cementum microleakage scores for the four groups. Kruskal-Wallis test value $=0.002$

\begin{tabular}{|c|c|c|c|c|c|}
\hline Group & 0 & 1 & 2 & 3 & 4 \\
\hline I & $13(65)$ & $3(15)$ & 1 (5) & $0(0)$ & $3(15)$ \\
\hline II & $11(55)$ & 1 (5) & 1 (5) & 3 (15) & $4(20)$ \\
\hline III & $2(10)$ & $0(0)$ & 1 (5) & $10(50)$ & 7 (35) \\
\hline IV & $9(45)$ & $2(10)$ & $2(10)$ & $3(15)$ & $4(20)$ \\
\hline Total & 35 (44) & $6(7)$ & $5(6)$ & $16(20)$ & 18 (23) \\
\hline
\end{tabular}


in microleakage has been demonstrated between two cylindrical class $\mathrm{V}$ cavities of different dimensions, but of the same $\mathrm{C}$-factor. ${ }^{15}$ Therefore, it was concluded that microleakage is more closely related to the volume of the restoration rather than to the $\mathrm{C}$-factor. ${ }^{14}$ Our results were very interesting, as class $\mathrm{V}$ cavities with higher $\mathrm{C}$-factor had more microleakage than class $\mathrm{V}$ cavities with lower $\mathrm{C}$ factor only when the fast curing mode was used. On the other hand, there was no difference in the amount of microleakage when the soft-start curing mode was used, regardless of the value of the $\mathrm{C}$-factor. In all groups, the volume of the restorations was the same. These results can be explained by the fact that fast curing mode produces higher stresses at the adhesive system, and these stresses have the worst effect in case of unfavorable cavity design (i.e. high $\mathrm{C}$-factor).

One could speculate that the variation between the results of different studies can be attributed to variations in methodology, for example, type of cavity prepared in each study (class I vs. class II vs. class V), type of teeth used (human vs. bovine vs. models), restorative materials used, the curing protocols employed in addition to the type of adhesive system and the way it has been manipulated. Another important factor is the way the investigators change the $\mathrm{C}$-factor of the cavity, i.e., by increasing the depth or the width of the cavity, as using cavities of different depths results in different dentinal properties, which can affect microleakage. In our study, we purposely changed the $\mathrm{C}$-factor by changing the shape of the cavities, keeping the volume and the depth of the cavities constant in all the tested groups.

One LED curing light was used in this study, but with two curing modes. Although the curing time was different between the two curing modes used, the total energy delivered was the same (16.5 J/ $\mathrm{cm}^{2}$ ). Previous studies demonstrated that softstart curing delivers low levels of energy initially, allowing the resin composite to flow. This releases the stresses of polymerization shrinkage, resulting in reducing microleakage. ${ }^{7,31,32}$ High polymerization stresses have been shown to increase leakage in class $\mathrm{V}$ cavities. ${ }^{12}$ On the contrary, Hofmann and Hunecke ${ }^{6}$ showed no difference between high intensity curing lights with soft-start curing, with regard to margin quality and marginal seal of class II resin composite restorations.
The use of the soft-start exponential mode of LED curing lights was found to reduce microleakage at both enamel and dentinal margins of class II cavities, when compared to the continuous light polymerization mode. ${ }^{23}$ In our study, the soft-start mode did not affect enamel microleakage in all of the groups, but it was able to reduce microleakage at the dentin/cementum margin only for high Cfactor cavities. It is difficult to compare our results with previous findings, as we prepared class $V$ cavities and other researchers used class II cavities. Other causes of difference could be related to the resin composite that was used and the placement technique followed in both studies.

Bonding of resins to dentin is far more difficult and less predictable than bonding to enamel. Dentin not only has a more complex histological structure than enamel, but also varies more with location. ${ }^{33}$ Although our results showed that the degree of enamel microleakage was less than that of dentin/cementum, this difference was not statistically different except for group III Iwhen the C-factor was high). In this group, the amount of microleakage at the dentinal/cementum margins was statistically higher than at the enamel margins. This result is supported by a previous study by Ozturk et al. ${ }^{25}$ In their study, they prepared box-shaped class $V$ cavities of the same dimensions used in the study at hand. They found higher microleakage at dentin/ cementum margins compared to enamel margins, regardless of the curing unit (conventional halogen curing unit, high intensity curing unit, and plasma arc curing unit). Clinically, clinicians should use the proper techniques to overcome this problem by manipulating the curing modes or by using reinmodified glass ionomer liners. ${ }^{3}$

The radiation exposure for all of the cavities was $16.5 \mathrm{~J} / \mathrm{cm}^{2}$. As we attempted to keep the volumes of the two types of cavities uniform, this resulted in differences in their surface areas. The box-shaped cavities had smaller surface areas, and therefore, their radiation exposure was less. Nonetheless, they had higher dye penetration values. Correction of the radiation exposure to correspond to the surface area is difficult to do in the settings of curing light used.

Clinically, research regarding the effect of softstart polymerization was equivocal. One study showed that it can reduce postoperative sensitivity following placement of resin composite restora- 
tions. ${ }^{2}$ On the other hand, another study demonstrated no effect of soft-start polymerization on postoperative sensitivity. ${ }^{34}$

It has been claimed that the predominant reason for reduced shrinkage stress, attained with soft-start polymerization, is the decrease in the final conversion of the resin composite, which can affect its mechanical properties. ${ }^{13}$ On the contrary, other studies have found that the degrees of conversion and microhardness of resin composite were not affected using various modes of LED curing lights. ${ }^{35-37}$ Previous research showed that soft-start curing to be effective in reducing microleakage. ${ }^{8,38}$ Our results showed that the fast-curing mode resulted in increased microleakage at the dentin/cementum margins of class $V$ cavities for box-shaped cavities, i.e., when the $\mathrm{C}$-factor was high. For V-shaped cavities, i.e., for low $\mathrm{C}$-factor cavities, there was no statistical difference between soft-start curing and fast-curing mode of the same curing light.

Traditionally, qualitative scoring systems were used to assess microleakage. Recently, with new advancements in image analysis software, quantitative microleakage measurements have become more popular. ${ }^{27,39}$ In this study, we compared the quantitative and qualitative scoring methods for microleakage to explore whether they produce different results. In the quantitative measurements we measured the actual degree of microleakage in micrometers, using special software to capture standardized images of the sections. While for the qualitative measurements we measured the degree of microleakage on a $0-4$ scale using the same images. Qualitative measurements could be done directly on the microscope without the need for special equipments. Both the quantitative measurements and the qualitative criteria gave the same results.

\section{CONCLUSIONS}

Under the conditions of this in vitro study, it can be concluded that:

- Using fast curing mode in class $V$ cavities with high $\mathrm{C}$-factor resulted in more microleakage at the dentin/cementum margins.

- In all the groups, microleakage was evident at both enamel and dentinal/cementum margins. And there was no statistical difference between the two margins except for group III (high $\mathrm{C}$-factor cavities and cured with the fast curing mode).

- Quantitative and qualitative methods for measuring microleakage gave the same results and can be used interchangeably.

\section{ACKNOWLEDGEMENT}

This research is supported by Kuwait University Grant No. DR 01/07.

\section{REFERENCES}

1. Yap AU, Wang HB, Siow KS, Gan LM. Polymerization shrinkage of visible light-cured composites. Oper Dent 2000;25:98-103.

2. Briso AL, Mestrener SR, Delicio G, Sundfeld RH, BedranRusso AK, de Alexandre RS, Ambrosano GM. Clinical assessment of postoperative sensitivity in posterior composite restorations Oper Dent 2007;32:421-426.

3. Alomari QD, Omar R, Akpata SE. Effect of LED curing modes on postoperative sensitivity after class II resin composite restorations. J Adhes Dent 2007;9:477-481.

4. Sakaguchi RL, Wiltbank BD, Murchison CF. Cure induced stresses and damage in particulate reinforced polymer matrix composites: a review of the scientific literature. Dent Mater 2005;21:43-46.

5. Ferracane JL. Developing a more complete understanding of stresses produced in dental composites during polymerization. Dent Mater 2005;21:36-42.

6. Hofmann N, Hunecke A. Influence of curing methods and matrix type on the marginal seal of class II resin-based composite restorations in vitro. Oper Dent 2006;31:97-105.

7. Ilie N, Felten K, Trixner K, Hickel R, Kunzelmann KH. Shrinkage behavior of a resin-based composite irradiated with modern curing units. Dent Mater 2005;21:483-489.

8. Kubo S, Yokota H, Yokota H, Hayashi Y. The effect of lightcuring modes on the microleakage of cervical resin composite restorations. J Dent 2004;32:247-254.

9. Leonard DL, Charlton DG, Roberts HW, Cohen ME. Polymerization efficiency of LED curing lights. J Esthet Restor Dent 2002; 14:286-295.

10. Lim BS, Ferracane JL, Sakaguchi RL, Condon JR. Reduction of polymerization contraction stress for dental composites by two-step light-activation. Dent Mater 2002;18:436-444.

11. Dewaele M, Asmussen E, Peutzfeldt A, Munksgaard EC, Benetti AR, Finné G, Leloup G, Devaux J. Influence of curing protocol on selected properties of light-curing polymers: degree of conversion, volume contraction, elastic modulus, and glass transition temperature. Dent Mater 2009;25:1576-1584. 
12. Fróes-Salgado NR, Pfeifer CS, Francci CE, Kawano Y. Influence of photoactivation protocol and light guide distance on conversion and microleakage of composite restorations. Oper Dent 2009;34:408-414.

13. Lu H, Stansbury JW, Bowman CN. Impact of curing protocol on conversion and shrinkage stress. J Dent Res 2005;84:822826.

14. Braga RR, Boaro LC, Kuroe T, Azevedo CL, Singer JM. Influence of cavity dimensions and their derivatives (volume and ' $C$ ' factor) on shrinkage stress development and microleakage of composite restorations. Dent Mater 2006;22:818-823.

15. Costa Pfeifer CS, Braga RR, Cardoso PE. Influence of cavity dimensions, insertion technique and adhesive system on microleakage of Class V restorations. J Am Dent Assoc 2006;137:197-202.

16. Duarte S Jr, Dinelli W, da Silva MH. Influence of resin composite insertion technique in preparations with a high $\mathrm{C}$ factor. Quintessence Int 2007;38:829-835

17. Feilzer, AJ, De Gee AJ, Davidson CL. Setting stress in composite resin relation to configuration of the restoration. $J$ Dent Res 1987;66:1636-1639.

18. Bouillaguet S, Ciucchi B, Jacoby T, Wataha JC, Pashley D. Bonding characteristics to dentin walls of class II cavities, in vitro. Dent Mater 2001;17:316-321.

19. Cunha LG, Alonso RC, Neves AC, de Goes MF, Ferracane JL, Sinhoreti MA. Degree of conversion and contraction stress development of a resin composite irradiated using halogen and LED at two C-factor levels. Oper Dent 2009;34:24-31.

20. Nikolaenko SA, Lohbauer $U$, Roggendorf $M$, Petschelt $A$, Dasch W, Frankenberger R. Influence of c-factor and layering technique on microtensile bond strength to dentin. Dent Mater 2004;20:579-585.

21. Davidson CL, de Gee AJ. Light-curing units, polymerization, and clinical implications. J Adhes Dent 2000;2:167-173.

22. Oberholzer TG, Schünemann M, Kidd M. Effect of LED curing on microleakage and microhardness of Class $\mathrm{V}$ resinbased composite restorations. Int Dent J 2004;54:15-20.

23. Nalcaci A, Ulusoy N. Effect of Thermocycling on microleakage of resin composites polymerized with LED curing techniques. Quintessence Int 2007;38:e433-439.

24. Oberholzer TG, Du Preez IC, Kidd M. Effect of LED curing on the microleakage, shear bond strength and surface hardness of a resin-based composite restoration. Biomaterials 2005;26:3981-3986.

25. Ozturk AN, Usumez A, Ozturk B, Usumez S. Influence of different light sources on microleakage of class $V$ composite restorations. J Oral Rehabil 2004;31:500-504.

26. Rosales-Leal JI. Microleakage of Class $V$ composite restorations placed with etch-and-rinse and self-etching adhesives before and after thermocycling. J Adhes Dent 2007;9:255-259
27. Yammazaki PCV, Bedran-Russo AKB, Pereira PNR, Swift Jr EJ. Microleakage evaluation of a new low-shrinkage composite restorative material. Oper Dent 2006;31:670-676.

28. Orellana N, Ramírez R, Roig M, Giner L, Mercade M, Durán F, Herrera G. Comparative study of the microtensile bond strength of three different total etch adhesives with different solvents to wet and dry dentin (in vitro test). Acta Odontol Latinoam 2009;22:47-56.

29. Watts DC, Marouf AS, Al-Hindi AM. Photo-polymerization shrinkage-stress kinetics in resin-composites: methods development. Dent Mater 2003;19:1-11.

30. Santini A, Ivanovic V, Ibbetson R, Milia E. Influence of cavity configuration on microleakage around Class $\mathrm{V}$ restorations bonded with seven self-etching adhesives. $J$ Esthet Restor Dent 2004;16:128-135; discussion 136.

31. Soh MS, Yap AU, Siow KS. Post-gel shrinkage with different modes of LED and halogen light curing units. Oper Dent 2004;29:317-324.

32. Yoshikawa T, Nakaoki Y, Takada T, Burrow MF, Tagami J. Effect of light-curing method and irradiation time on marginal sealing and cavity wall adaptation of resin composite restorations. Am J Dent 2003;16:63A-67A.

33. Swift Jr EJ. Dentin/enamel adhesives: review of the literature. Pediatr Dent 2002;24:456-461.

34. Chan DC, Browning WD, Frazier KB, Brackett MG. Clinical evaluation of the soft-start (pulse-delay) polymerization technique in Class I and II composite restorations. Oper Dent 2008;33:265-271.

35. Alomari QD, Mansour YF. Effect of LED curing modes on cusp deflection and hardness of composite restorations. Oper Dent 2005;30:684-689.

36. Felix CA, Price RB, Andreou P. Effect of reduced exposure times on the microhardness of 10 resin composites cured by high-power LED and QTH curing lights. J Can Dent Assoc $2006 ; 72: 147$.

37. Tarle Z, Knezevic A, Demoli N, Meniga A, Sutaloa J, Unterbrink G, Ristic M, Pichler G. Comparison of composite curing parameters: effects of light source and curing mode on conversion, temperature rise and polymerization shrinkage. Oper Dent 2006;31:219-226.

38. Nalçaci A, Salbas M, Ulusoy N. The effects of soft-start vs continuous-light polymerization on microleakage in class II resin composite restorations. J Adhes Dent 2005;7:309-314.

39. Cehreli ZC, Gungor HC. Quantitative microleakage evaluation of fissure sealants applied with or without a bonding agent: results after four-year water storage in vitro. $J$ Adhes Dent 2008;10:379-384. 